\title{
Informationsverhalten
}

\author{
Philipp Liebrenz*
}

\section{Das Usability Lab am Schweizerischen Institut für Informationswissenschaft}

https://doi.org/10.1515/iwp-2020-2117

Google plant neu die User Experience stärker bei der Relevanzberechnung von Webseiten zu bewerten. Doch neu ist das Thema User Experience nicht. Das Schweizerische Institut für Informationswissenschaft (SII) beschäftigt sich seit nunmehr über 15 Jahren mit Fragestellungen aus diesem Bereich. Das SII betreibt zudem seit vielen Jahren erfolgreich eines der am besten ausgestatteten Usability Labore der Schweiz.

Ende Mai 2020 hat Google angekündigt, die Page Experience bei der Berechnung der Webseitenrelevanz für die Google Suche noch stärker einzubeziehen (Subramanian, 2020). Ein klares Statement für die Wichtigkeit von User Experience bei Webseiten. In der Ankündigung von Google wird aufgeführt, dass neben Ladezeit und Mobiltauglichkeit weitere Metriken in das Ranking der Webseiten integriert werden sollen, die die Qualität des Nutzungserlebnisses von Webseiten bewerten. Damit entscheidet die User Experience zukünftig noch stärker mit, ob eine Webseite im Web gefunden wird und das Nutzererlebnis wird damit einmal mehr zum Erfolgsfaktor von Webseiten.

\section{Was ist User Experience?}

Mit User Experience bezeichnet man das Erlebnis, das Nutzende beim Gebrauch eines Produkts erfahren. Es umfasst die Aspekte der eigentlichen Nutzung (z.B. Gebrauchstauglichkeit oder engl. Usability, Joy-of-Use) aber auch vor- und nachgelagerte Aspekte des Erlebnisses (z. B. Erfahrungen mit der Marke, Supportanfragen, ...). Damit ein Produkt erfolgreich ist, müssen diese Erlebnisse positiv sein. Im Allgemeinen bewerten Menschen ein Erlebnis dann positiv, wenn ihre Bedürfnisse erfüllt oder gar übertroffen wurden.

*Kontaktperson: Philipp Liebrenz, MSc, Fachhochschule Graubünden, Schweizerisches Institut für Informationswissenschaft, Pulvermühlestrasse 57, 7000 Chur, Schweiz,

E-Mail: philipp.liebrenz@fhgr.ch
Die systematische Erarbeitung der User Experience passiert im User-centred Design-Prozess. Dabei stehen die Nutzenden und ihre Bedürfnisse im Mittelpunkt des Entwicklungsprozesses. Zu Beginn werden Angaben über die Nutzenden, den Nutzungskontext des Produkts und die Ziele der Nutzenden systematisch erhoben. Ausgehend von diesen Daten werden Ideen und Konzepte entwickelt und in Prototypen greifbar gemacht. Zuletzt wird validiert, inwieweit die erarbeiteten Lösungen den Bedürfnissen der Nutzenden entsprechen. Gegebenenfalls wird der Prozess iteriert. Dieser Ansatz ermöglicht innovative, begehrenswerte Produkte, die nicht auf der grünen Wiese entwickelt wurden, sondern auf tatsächlichen Bedürfnissen und Erwartungen der Nutzenden beruhen.

\section{Das Usability Labor der FHGR}

Seit nunmehr 15 Jahren beschäftigt sich eine Arbeitsgruppe am SII mit Fragestellungen rund um das Thema User Experience und User-centred Design. Dreh- und Angelpunkt dieser Aktivitäten ist ein umfassend ausgestattetes Usability-Labor. Es verfügt über verschiedenste Aufzeichnungsgeräte zur Analyse und Dokumentation von Nutzungsverhalten bei digitalen Produkten. Hierzu gehören ein Beobachtungsraum, ein Testraum mit festinstallierten Kameras, verschiedene Eyetracker (ein Mobile Eyetracker, ein stationärer Eyetracker, eine Brille mit Eyetracking und eine VR Brille mit Eyetracking) sowie verschiedene spezialisierte Softwareanwendungen. Durch die Eyetracker werden, zum Beispiel, Blickbewegungen von Testpersonen erfasst und dokumentiert. Dadurch lässt sich analysieren, welche Bereiche einer Softwareanwendung besonders häufig oder intensiv betrachtet werden. Diese Erkenntnisse dienen dazu, die Benutzeroberflächen zu optimieren und Schwachstellen zu identifizieren. 


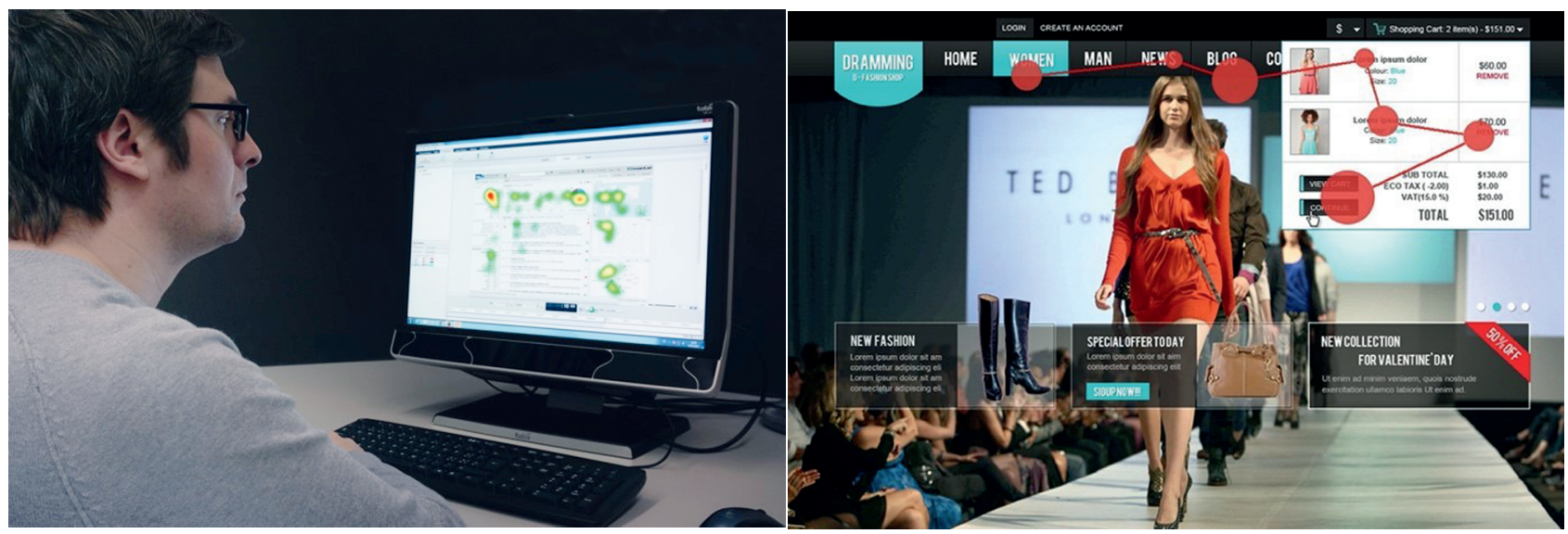

Abbildung 1 und 2: Beispielhafte Darstellung der Anwendungsmöglichkeiten von Eyetracking-Studien. Links Auswertung der Blickpunkte in Form einer Heatmap (Bild: SII). Rechts sind die Reihenfolge und Dauer der Betrachtung einzelner Elemente bei der Betrachtung einer Ecommerce Webseite dargestellt. (Bild und Copyright @ 2020 Tobii AB)

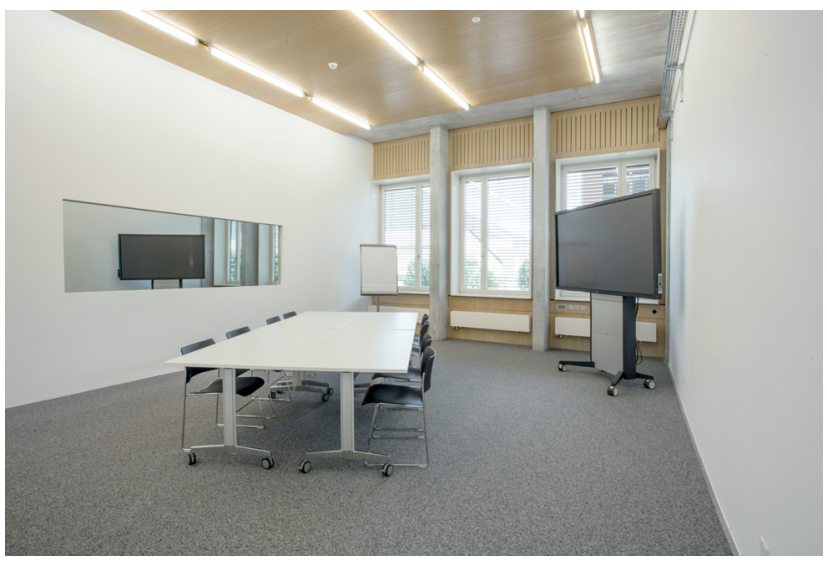

Abbildung 3: Das Usability Labor an der FHGR. Durch den einseitig durchsichtigen Spiegel können Testpersonen bei Usability Tests beobachtet werden.

Das Usability Labor ist darauf ausgerichtet Unternehmen und öffentliche Institutionen bei der Evaluation von Benutzeroberflächen zu unterstützen und zu beraten. In Usability Tests werden Benutzer mit einem Prototyp oder fertigen Produkt konfrontiert und gebeten Aufgaben zu erledigen. Durch Befragungen und Beobachtungen lassen sich Rückschlüsse auf Stärken und Schwächen des Produkts ziehen, um mögliche Verbesserungsvorschläge $\mathrm{zu}$ erarbeiten.

Neben der Evaluation von Benutzeroberflächen bietet das Usability Labor auch Dienstleistungen entlang des User-centred Design-Prozesses an. Das Team des Labors unterstützt Unternehmen auch bei Erforschung von Nutzerbedürfnissen, bei der Erstellung erster Konzepte sowie der Gestaltung und Implementierung von Prototypen und der anschließenden Evaluation. In diesem Zusammenhang durfte das Usability Labor in den vergangenen Jah- ren viele namenhafte Unternehmen bei der Entwicklung von Konzepten und Prototypen unterstützen.

Aber auch in Lehre und Forschung engagiert sich das Usability Labor. So hat es beispielsweise im Programm Wissenschaftliche Information im Auftrag von swissuniversities bei der Sicherstellung der Gebrauchstauglichkeit im Rahmen verschiedenster Projekte mitgewirkt. In der Lehre wird den Studierenden die Funktionsweise der Apparaturen des Labors aufgezeigt und die Studierenden werden in den Methoden des User-centred Design-Prozesses geschult.

\section{Remote Usability Testing}

Die Aktivitäten des Teams beschränkten sich nicht nur auf das physische Labor. Ein weiteres Angebot des Teams ist das Remote Usability Testing.

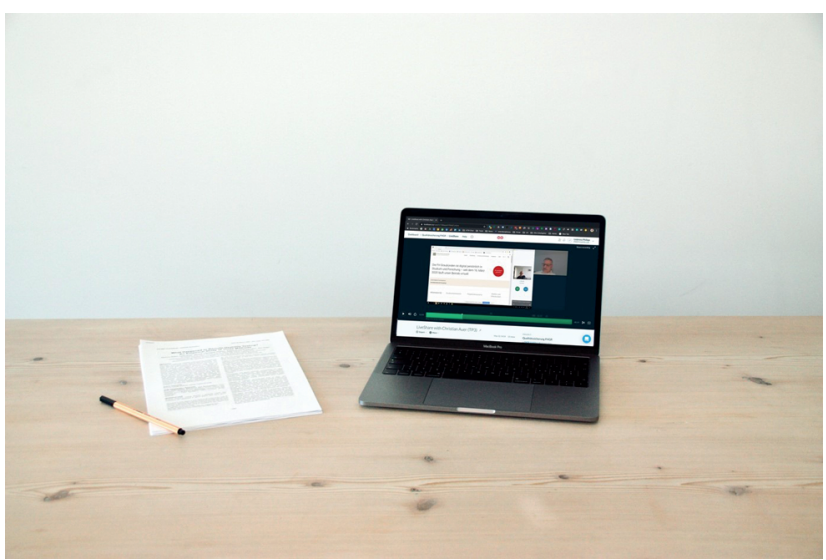

Abbildung 4: Remote Usability Testing benötigt nur wenige technische Gerätschaften im Vergleich zum Labortest. (Bild: SII) 
Beim Remote Usability Testing wird der herkömmliche Usability Test vom Labor in das Web verlegt. Die Probandinnen und Probanden sowie die Testleitung sind demzufolge räumlich voneinander getrennt. In der Vergangenheit wurde diese Methode meist verwendet, wenn die Testpersonen schwer erreichbar waren oder viele Personen getestet werden sollten.

Durch Remote Testing können Ressourcen eingespart werden. In Zeiten der Coronavirus-Pandemie ist zudem von Vorteil, dass kein physischer Kontakt besteht und es daher kein Ansteckungsrisiko gibt. Außerdem sind die Testpersonen während des Remote Tests in ihrem natürlichen Umfeld. Dadurch wird die künstliche Labor- und Testsituation abgemildert, sodass mit valideren Ergebnissen gerechnet werden kann. Nachteile, wie bei jedem Test außerhalb des Labors, sind allerdings unter anderem die geringere Kontrolle auf die Einflussfaktoren, die mögliche Ablenkung der Nutzenden oder Probleme mit dem technischen Setup.

Auch im Bereich Remote Usability Testing konnte das Usability Lab bereits Erfahrungen sammeln und Projekte durchführen. So wurden im Auftrag eines Spitals Interviews zu einer Webseite durchgeführt und analysiert.

Deskriptoren: Usability Labor, Nutzer, Benutzerforschung Fachhochschule, Schweiz

\section{Links}

FHGR Usability Labor, https://www.fhgr.ch/forschung-u nd-dienstleistung/labore/usability-lab/

\section{Literatur}

Hartson, H. R., Castillo, J. C., Kelso, J. \& Neale, W. C. (1996). Remote evaluation: the network as an extension of the usability laboratory. In: Proceedings of the SIGCHI conference on Human factors in computing systems common ground - $\mathrm{CHI}$ '96. Vancouver, British Columbia, Canada: ACM Press. 1996. S. 228-235. ISBN 978-0-89791-777-3, http://portal.acm.org/citation.cfm?d oid=238386.238511 [14.7.2020].

Sauer, J., Sonderegger, A., Heyden, K., Biller, J., Klotz, J. \& Uebelbacher, A. (2019). Extra-laboratorial usability tests: An empirical comparison of remote and classical field testing with lab testing. Applied Ergonomics. 1 Januar 2019. Bd. 74, S. 85-96. DOI 10.1016/j.apergo.2018.08.011.

Schade, A. (2013). Remote Usability Tests: Moderated and Unmoderated. Nielsen Norman Group [online]. 12 Oktober 2013. https:// www.nngroup.com/articles/remote-usability-tests/ [14.7.2020].

Subramanian, S. (2020). Evaluating page experience for a better web. https://webmasters.googleblog.com/2020/05/evaluat ing-page-experience.html [14.7.2020].

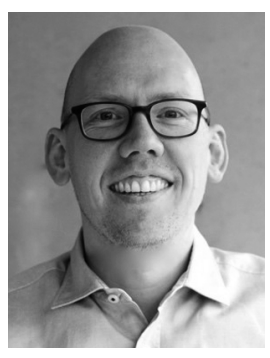

Philipp Liebrenz, MSc

Fachhochschule Graubünden

Schweizerisches Institut für

Informationswissenschaft

Pulvermühlestrasse 57

7000 Chur

Schweiz

philipp.liebrenz@fhgr.ch

Philipp Liebrenz ist Dozent für User Experience am Schweizerischen Institut für Informationswissenschaft (SII). Er studierte Information Engineering an der Universität Konstanz und Human-Computer Interaction an dem University College London. Nach dem Studium arbeitete er unter anderem als User Experience Designer bei Vodafone, als User Experience Researcher bei Samsung, als Usability Consultant bei der UBS und war als Interaction Designer und Manager in verschiedenen Bereichen bei der Swisscom tätig. 